\title{
Do lifestyle interventions affect dietary diversity score in the general population?
}

\author{
Nizal Sarrafzadegan ${ }^{1, *}$, Leila Azadbakht ${ }^{2}$, Noushin Mohammadifard', Ahmad \\ Esmaillzadeh $^{2}$, Morteza Safavi ${ }^{2,3}{ }^{\text {, Firoozeh Sajadi }}{ }^{1}$, Hasan Alikhasi ${ }^{1}$, Maryam \\ Maghroun ${ }^{1}$ and Roya Kelishadi ${ }^{1}$ \\ 'Isfahan Cardiovascular Research Center (WHO Collaborating Center in the Eastern Mediterranean Region), \\ Isfahan University of Medical Sciences, PO Box 81465-1 148, Isfahan, Iran: ${ }^{2}$ Department of Nutrition, School of \\ Public Health, Isfahan University of Medical Sciences, Isfahan, Iran: ${ }^{3}$ Community Nutrition Department, Ministry \\ of Health and Medical Education, Tehran, Iran
}

Submitted 17 June 2008: Accepted 24 November 2008: First published online 23 February 2009

\begin{abstract}
Objective: The dietary diversity score (DDS) is a good indicator of diet quality as well as of diet-disease relationships; therefore, the present study was undertaken to reveal the effect of a lifestyle intervention on this index.

Design: A baseline and three evaluation studies were conducted in two intervention districts (Isfahan and Najaf-Abad) and a reference area (Arak), all located in central Iran. The Isfahan Healthy Hearth Programme (IHHP) targeted the entire population of nearly 2 million in urban and rural areas of the intervention communities. One of the main strategies of the lifestyle intervention phase in the IHHP was healthy nutrition. Usual dietary intake was assessed using a fortynine-item FFQ. A diversity score for each food group was calculated and the DDS was considered the sum of the diversity scores of the food groups.

Results: There were significant increases in DDS in both intervention areas $(P=0 \cdot 0001)$ after controlling for confounding factors. There was a significant interaction between area and evaluation stage with regard to DDS $(P=0 \cdot 0001)$. The effect of the intervention on the diversity scores of all food groups was also significant $(P=0.0001$ for all) after adjusting for socio-economic status.

Conclusion: The community-based lifestyle intervention in the IHHP was successful in improving DDS which might be related to an increase of diet quality of the population that in turn might decrease the risks of chronic diseases.
\end{abstract}

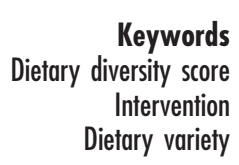

Moving from a monotonous diet to a more diverse diet has been shown to increase energy and micronutrient intakes in developing countries ${ }^{(1-5)}$. Intake of a diverse range of foods has been a recommendation for achieving adequate nutrient intake and such advice appears in the dietary guidelines of many countries ${ }^{(6)}$. The most recent dietary guidelines recommend using a variety of whole grains, fruits and vegetables ${ }^{(7)}$. There is an increasing trend towards assessing the nutrient quality of the $\operatorname{diet}^{(7)}$. Especially in developing countries, methods for evaluating nutrient adequacy should be simple and practical ${ }^{(8)}$. Previous studies in Iran have shown that a dietary diversity score (DDS), as well as the diversity score of each separate food group, is associated with diet quality in adult men, women and adolescents ${ }^{(4,5,9)}$

Furthermore, assessing overall diet is more informative than looking at only one single nutrient. According to previous studies, higher DDS is associated with greater intakes of fibre ${ }^{(2,4)}$ as well as vitamin $\mathrm{C}^{(2)}$ and $\mathrm{Ca}^{(4)}$. These nutrients have a negative association with CVD, hypertension and obesity $^{(10-12)}$. There are several studies that show a relationship between diet variety and mortality ${ }^{(13)}$, cancer $^{(14)}$, cardiovascular health $^{(15-17)}$ and metabolic syndrome ${ }^{(18)}$.

The intervention phase in the Isfahan Healthy Heart Programme (IHHP) was conducted to improve lifestyle behaviours including dietary habits, physical activity level, tobacco control and stress management at community level ${ }^{(19)}$. Along with improving nutritional status, we wondered if we could assess the whole diet by determining the DDS before and after the intervention. As the DDS is a good indicator of diet quality as well as diet-disease relationships, the present study was undertaken to reveal whether or not the DDS was changed by the lifestyle intervention.

\section{Methods}

\section{Population}

The study design and rationale of the IHHP and intervention methods have been described elsewhere ${ }^{(20)}$. 
Briefly, two intervention districts (Isfahan and NajafAbad) and a reference area (Arak), all located in central Iran, were included in the study. According to the 2000 National Census, the population was 1895856 in Isfahan and 275084 in Najaf-Abad, a small district neighbouring Isfahan. Arak, located $375 \mathrm{~km}$ north-west of Isfahan with a population of 668531, was selected as reference area because of similarities to the intervention areas in terms of socio-economic, demographic and health profile and good cooperation ${ }^{(20)}$. The intervention programme targeted the whole population in urban and rural areas of the intervention communities. Arak was monitored for evaluation purposes but did not receive interventions. Measurements were done at baseline and annually for four years in the intervention areas and three years in the reference area. Surveys were performed in the same years in both the intervention and control areas. A given number of individuals (independent sample surveys) from among the residents of the whole community were randomly selected by a multistage cluster sampling method in each annual evaluation. The sample studied in every survey was different because this was a populationbased study and the sample size for each survey was calculated by a statistician in order that this sample would be a representative sample of the society. Informed written consent was provided by each participant. The study was approved by the research council and the ethical committee of the Isfahan Cardiovascular Research Center of Isfahan University of Medical Sciences.

\section{Interventions}

The intervention programme targeted the general population as well as specific target groups in urban and rural areas of the intervention communities. Key strategies for intervention activities included public education through mass media, inter-sectoral cooperation and collaboration, professional education and involvement, marketing and organizational development, legislation and coordination, and policy development, as well as research and evaluation. The main factors targeted by IHHP were healthy nutrition, increased physical activity, tobacco control and stress management. Interventions were targeted to individuals, populations and the environment based on results obtained from the baseline surveys and needs assessment, as well as existing health services. The programme comprised different projects on women, children, adolescents, high-risk groups and cardiac patients. An underlying principle in all ten projects was to develop and maintain close contact with representatives of relevant community organizations. The teams worked intensively and closely with representatives of mass media (television, newspapers, radio, etc.), health professionals (administrators, physicians, nurses, health workers and volunteers, social workers, school staff, etc.), business and market leaders (food industry, groceries, bakeries, fast-food shops), key staff in non-governmental organizations and local political decision makers (county, municipal and provincial leaders). Details of the interventions as well as IHHP organization are described elsewhere ${ }^{(21)}$.

The 'Healthy Foods for Healthy Community Project' was one of the main projects of the IHHP. Many activities were performed for improving the food habits in society, including the training of kitchen staff in factories and improving the preparation and distribution of food in restaurants, sandwich sellers, pizzerias, and offices and organizations that serve food to their own personnel. Other activities were offering suitable strategies for the production of healthy food products, such as low-salt high-fibre bread, low-fat low-sugar candy and confections and low-fat dairy products; encouraging hydrogenated oil factories to produce oil with lower saturated and trans fatty acids; cooperating with the commerce organization to increase coupon-based distribution of liquid oil; and improving the labelling of food products. Educational materials such as books, compact disks, educational brochures and leaflets were also published and distributed in the intervention area ${ }^{(22)}$.

\section{Measurements}

Measurements were done at baseline and annually for up to four years in the intervention areas and up to three years in the reference area. Measurements in the fourth year have not yet been done in the reference area because of budget insufficiency. Usual dietary intake was assessed using a forty-nine-item FFQ adapted from the validated Countrywide Integrated Non-communicable Disease Intervention (CINDI) programme. All questionnaires were administered by trained dietitians. The FFQ consists of a list of foods commonly consumed by Iranians (Appendix). Participants were asked to report their frequency of consumption and common portion sizes of each food item during the previous year on a daily (e.g. bread), weekly (e.g. rice, meat) or monthly (e.g. fish) basis. The reported frequency for each food item was then converted to a daily intake.

Other variables such as age, sex, smoking behaviour, place of residence, socio-economic status and educational level were collected by using validated questionnaires.

\section{Dietary diversity score}

First, we divided foods into six groups and then calculated the diversity score of each group. To be counted as a 'consumer' for any of the food group categories, a respondent needed to consume at least one-half serving per day as defined by the Food Guide Pyramid quantity criteria $^{(2)}$; otherwise this score was considered zero. Finally, for calculating the diversity of each food group, we considered the sum of scores of the total subgroups divided by the number of subgroups in each food group and then multiplied by 2 . So the diversity score of each food group could be between 0 and 2. For calculating the DDS, the sum of the diversity scores of the six food groups (grain diversity score, dairy diversity score, fruit 
diversity score, vegetable diversity score, meat diversity score and oil diversity score) was considered. Hence, the DDS ranged between 0 and 12 .

\section{Statistical methods}

Statistical analysis was performed using the SPSS for Windows statistical software package version $13 \cdot 0$ (SPSS Inc., Chicago, IL, USA). The trend of the DDS and the diversity score for each food group were analysed by two-way ANONA separately in the three areas and at the different evaluation stages. The effect of socio-economic status, age, residency and literacy was adjusted when the means are reported. In these analyses, the $P$ values for area, evaluation stage and also the interaction between area and evaluation stage are presented separately.

DDS was divided into three categories $(<50 \%$ of the maximum score of DDS; between $50 \%$ and $75 \%$ of the maximum score of DDS; $>75 \%$ of the maximum score of the DDS), thus DDS was categorized as a score of $<6,6-9$ or $>9$. Besides this category, the quartiles of DDS were also determined. The $\chi^{2}$ test was used to compare the prevalence of the population in different quartiles of DDS and the three categories of DDS at baseline and after intervention in all three areas.

\section{Results}

General characteristics of the study participants are shown in Table 1. There were no significant differences in mean age, sex distribution or response rate between the intervention and reference areas. Table 2 shows the results of the multivariate-adjusted mean DDS across the different evaluation stages of the intervention and different areas of

Table 1 General characteristics of the study populations participating in the annual evaluations: Isfahan Healthy Heart Programme, Iran

\begin{tabular}{|c|c|c|c|c|c|c|c|c|c|c|c|}
\hline & & & \multicolumn{8}{|c|}{ Annual evaluation } & \multirow[b]{2}{*}{$P$} \\
\hline & \multicolumn{2}{|c|}{ Baseline } & \multicolumn{2}{|c|}{ First } & \multicolumn{2}{|c|}{ Second } & \multicolumn{2}{|c|}{ Third } & \multicolumn{2}{|c|}{ Fourth } & \\
\hline \multirow[t]{2}{*}{ No. of intervention/control subjects } & \multicolumn{2}{|c|}{$6175 / 6339$} & \multicolumn{2}{|c|}{$2994 / 2897$} & \multicolumn{2}{|c|}{$2400 / 2393$} & \multicolumn{2}{|c|}{$3012 / 3070$} & \multicolumn{2}{|c|}{$3011 /-$} & \\
\hline & Mean & $\mathrm{SD}$ & Mean & SD & Mean & SD & Mean & $\mathrm{SD}$ & Mean & SD & \\
\hline \multicolumn{12}{|l|}{ Age (years) } \\
\hline Intervention area* & $38 \cdot 6$ & $14 \cdot 7$ & $40 \cdot 4$ & $15 \cdot 4$ & $40 \cdot 7$ & $15 \cdot 6$ & $45 \cdot 1$ & $17 \cdot 3$ & $45 \cdot 6$ & $17 \cdot 3$ & $<0.01$ \\
\hline \multirow[t]{2}{*}{ Reference areat } & $39 \cdot 1$ & $15 \cdot 1$ & $40 \cdot 5$ & $15 \cdot 3$ & $40 \cdot 5$ & $15 \cdot 9$ & $45 \cdot 0$ & $17 \cdot 3$ & - & & $<0.01$ \\
\hline & \multicolumn{2}{|c|}{$\%$} & \multicolumn{2}{|c|}{$\%$} & \multicolumn{2}{|c|}{$\%$} & \multicolumn{2}{|c|}{$\%$} & \multicolumn{2}{|l|}{$\%$} & \\
\hline \multicolumn{12}{|l|}{ Females } \\
\hline Intervention area & \multirow{2}{*}{\multicolumn{2}{|c|}{$\begin{array}{l}51 \cdot 3 \\
50 \cdot 8\end{array}$}} & \multirow{2}{*}{\multicolumn{2}{|c|}{$\begin{array}{l}50 \cdot 0 \\
51 \cdot 0\end{array}$}} & \multirow{2}{*}{\multicolumn{2}{|c|}{$\begin{array}{l}50 \cdot 3 \\
50 \cdot 5\end{array}$}} & & & $51 \cdot 4$ & & $>0.05$ \\
\hline Reference area & & & & & & & & & - & & $>0.05$ \\
\hline Response rate & & & & & & & & & & & \\
\hline Intervention area & 9 & & 9 & & 10 & & 10 & & 100 & & - \\
\hline Reference area & 10 & & 9 & & 95 & & 10 & & - & & - \\
\hline Place of residence (\% rural) & & & & & & & & & & & \\
\hline Intervention area & & & & & & & & & $11 \cdot 9$ & & $<0.01$ \\
\hline Reference area & & & & & & & & & - & & $<0.05$ \\
\hline Illiterate & & & & & & & & & & & \\
\hline Intervention area & & & & & & & & & $21 \cdot 0$ & & $<0.01$ \\
\hline Reference area & & & & & & & & & - & & $<0.01$ \\
\hline Income level & & & & & & & & & & & \\
\hline Intervention area & & & & & & & & & & & \\
\hline Low incomeł & & & & & & & & & $21 \cdot 1$ & & $<0.01$ \\
\hline Moderate income & & & & & & & & & $76 \cdot 8$ & & $<0.01$ \\
\hline High incomell & & & & & & & & & $2 \cdot 1$ & & $<0.01$ \\
\hline Reference area & & & & & & & & & & & \\
\hline Low income & & & & & & & & & - & & $<0.01$ \\
\hline Moderate income & & & & & & & & & - & & $<0.01$ \\
\hline High income & & & & & & & & & - & & $<0.01$ \\
\hline Marital status & & & & & & & & & & & \\
\hline Intervention area & & & & & & & & & & & \\
\hline Married & & & & & & & & & $77 \cdot 7$ & & $<0.01$ \\
\hline Single & & & & & & & & & $12 \cdot 7$ & & $<0.01$ \\
\hline Divorced & & & & & & & & & 0.6 & & $<0.01$ \\
\hline Reference area & & & & & & & & & & & \\
\hline Married & & & & & & & & & - & & $<0.01$ \\
\hline Single & & & & & & & & & - & & $<0.01$ \\
\hline Divorced & & & & & & & & & - & & $<0.01$ \\
\hline
\end{tabular}

*Najafabad and Isfahan were the intervention areas.

tArak was the reference (control) area.

tLow income: $<1000000 R$.

\$Moderate income: $1000000-<5000000 R$.

IHigh income: $>5000000 R$. 
Table 2 Multivariate-adjusted mean dietary diversity score (DDS) across the different evaluation stages of the intervention and different areas of the study: Isfahan Healthy Heart Programme, Iran

\begin{tabular}{|c|c|c|c|c|c|c|c|c|c|c|c|}
\hline & \multicolumn{2}{|c|}{ Najafabad* } & \multicolumn{2}{|c|}{ Isfahant } & \multicolumn{2}{|c|}{ Najafabad and Isfahan } & \multicolumn{2}{|c|}{ Arak‡ } & \multicolumn{3}{|c|}{$P \S$} \\
\hline & Mean & $\mathrm{SD} \uparrow$ & Mean & SD & Mean & SD & Mean & SD & Area & Evaluation stage & Area $\times$ evaluation stage \\
\hline DDS $\|$ & & & & & & & & & 0.0001 & 0.0001 & 0.0001 \\
\hline Baseline & 1.09 & 0.02 & $1 \cdot 34$ & 0.01 & $1 \cdot 22$ & 0.02 & $1 \cdot 45$ & 0.01 & & & \\
\hline First evaluation** & $1 \cdot 11$ & 0.03 & $1 \cdot 47$ & 0.02 & $1 \cdot 30$ & 0.02 & $1 \cdot 31$ & 0.61 & & & \\
\hline Second evaluationtt & $1 \cdot 30$ & 0.03 & 1.55 & 0.02 & $1 \cdot 42$ & 0.03 & $1 \cdot 31$ & 0.01 & & & \\
\hline Third evaluationłł & $2 \cdot 74$ & 0.02 & $2 \cdot 55$ & 0.02 & $2 \cdot 65$ & 0.03 & $1 \cdot 31$ & 0.01 & & & \\
\hline
\end{tabular}

*Najafabad was an intervention area; number of subjects in Najafabad at baseline, first evaluation, second evaluation and third evaluation was $1988,896,720$ and 1008, respectively.

†lsfahan was an intervention area; number of subjects in Isfahan at baseline, first evaluation, second evaluation and third evaluation was $4187,2098,1680$ and 2004, respectively.

$\ddagger$ Arak was the reference area; number of subjects in Arak at baseline, first evaluation, second evaluation and third evaluation was $6339,2897,2393$ and 3071 , respectively.

$\S P$ values from two-way ANOVA.

IIDDS is the sum of the diversity scores of six food groups (grain diversity score, dairy diversity score, fruit diversity score, vegetable diversity score, meat diversity score and oil diversity score).

-Estimated marginal means and standard deviations adjusted for socio-economic status, age, residency and literacy (all such values).

** The first evaluation was conducted after 1 year of the study.

$t+T$ The second evaluation was conducted after 2 years of the study.

$\$ \neq$ The third evaluation was conducted after 3 years of the study.

the study. There was a jump in DDS between the second and third evaluation time points. After controlling for socio-economic status, there were significant increases in DDS in Najafabad and Isfahan (intervention areas) during the intervention (area main effect: $P=0 \cdot 0001$ for all, twoway ANOVA; evaluation stage main effect: $P=0 \cdot 0001$ for all, two-way ANOVA). There was a significant interaction between area and evaluation stage with regard to DDS ( $P=0 \cdot 0001$, two-way ANOVA). Table 3 shows multivariate-adjusted mean diversity scores of food groups across the different evaluation stages of the intervention and different areas of the study. The diversity scores of all food groups changed significantly during the different evaluation stages of the intervention. There was a very large increase in the dairy diversity score in the intervention areas between the second and third evaluation. A significant interaction between area and evaluation stage with regard to each diversity score of food groups was seen $(P=0 \cdot 0001$, two-way ANOVA).

At baseline, all of the population in the three areas under study had a DDS of $<6$ (50\% of the maximum score of DDS). Even after 3 years of intervention, this prevalence reduced to $98 \%$ and only $2 \%$ of the population had DDS score of 6-9 (between $50 \%$ and $75 \%$ of the maximum score of DDS). When we looked at the DDS quartiles, almost all of the population before and after the intervention was in the first and second quartiles (data not shown).

\section{Discussion}

The results of the present study show that communitybased lifestyle interventions in the IHHP were successful in increasing the DDS as well as the diversity score of each food group. This increasing trend in DDS and diversity scores of food groups was significant for both area and evaluation stage, separately. While the DDS and all diversity scores of food groups were increased in the intervention areas (Najafabad and Isfahan), the DDS, diversity score of grain, diversity score of fruit and diversity score of vegetables decreased significantly in the reference area (Arak). We do not have any logical interpretation for this reduction but may be that the increasing price of food items is the reason, which did not affect the intervention area because of nutritional intervention.

Recently, researchers have shifted their focus from a nutrient-based approach to the whole dietary intake. By analysing the dietary intake of the population, the potential effect of known and unknown interactions among foods and nutrients may be taken into account. Furthermore, from a public health perspective, indices of the whole diet assessment such as the DDS may present a more precise judgement than looking at the nutrient intake per $\mathrm{se}^{(23)}$. The present study provided an opportunity to evaluate the diet by using a whole dietary assessment index before and after a lifestyle intervention. In this case, we were able to evaluate the impact of the intervention not only on nutrient consumption but also on dietary intake. Most previous nutritional interventions have focused on the effect of intervention on the intake of specific nutrients or the prevalence of chronic diseases and their risk factors ${ }^{(24-27)}$. To the best of our knowledge, the present study is the first one reporting the effect of lifestyle intervention on DDS.

There was a jump in DDS between the second and third evaluation which may be due to passing two phases of intervention and increasing the duration of nutritional intervention after two intervention phases. It seems that people got more familiar with the concepts of the intervention after the passage of more time. In particular, there was a large increase in the dairy diversity score in the intervention areas between the second and third evaluation which may be due to nutritional education 
of the public through television and industry producing more kinds of dairy products, especially the low-fat versions

Previous studies showed that dietary variety can be a good indicator of nutrient adequacy ${ }^{(2-7)}$. Thus the trend in the present intervention, of increasing DDS after five years of nutritional intervention at community level, could be an indicator of increasing dietary adequacy in the intervention areas.

Two reports regarding the diversity score of food groups and special nutrient adequacy also showed how different food group varieties contribute to the probability of nutrient adequacy among a representative group of Tehranian adults ${ }^{(5,9)}$. It has been mentioned that variety of whole grains is associated with protein and vitamin $\mathrm{B}_{2}$ intakes and the variety of whole grains may be used as a simple method for evaluating the adequacy of the mentioned nutrients ${ }^{(5,9)}$. Therefore, the increasing diversity score of grains during the intervention period in the present study may be an indicator of increasing protein and vitamin $B_{2}$ intakes among the people in the intervention areas. This might be due to increasing the consumption of whole grains, which was one of the aims in the IHHP nutrition project. Regarding the association between DDS and chronic diseases, previous studies have shown an inverse association between DDS and metabolic risks which may be attributed to the higher consumption of healthier food groups associated with higher DDS. Subjects who had higher DDS consumed more fibre, fruit, vegetables and vegetable oil ${ }^{(13-18)}$. Therefore, increased DDS after the lifestyle intervention in the present study indicates that this intervention might result in a lower prevalence of risk factors for chronic diseases. However, chronic diseases such as the metabolic syndrome and CVD are heterogeneous and besides dietary pattern, other factors such as hereditary factors may need to be considered. Additionally, most of the risk factors are interrelated and this could confound the relationship between DDS and metabolic risk factors.

Our findings need to be interpreted while considering some limitations. We used an FFQ to assess dietary intakes. So, misclassification is a major concern in our study, as it is in all epidemiological studies. When we categorized subjects according to DDS quartiles, all of the population was in the first and second quartiles. This could, to some extent, be explained by an inadequate number of food items in the FFQ to cover all the possible food items in each food group. In the present study, we adjusted for the effect of socio-economic status in reporting the mean values for DDS and diversity scores of food groups, which might be a strength point.

In conclusion, the lifestyle intervention in the IHHP, reported in the present study, was successful in improving DDS which might be related to an increase of diet quality of the population that in turn might decrease the risks of chronic diseases.

\section{Acknowledgements}

The study was supported by financially by Isfahan University of Medical Sciences and Isfahan Cardiovascular Research Center. None of the authors has any personal or financial conflicts of interest. L.A. and A.E. analysed the data and wrote the manuscript. N.M., M.S., F.S., H.A. and M.M. conducted and collected the data. N.S. and R.K. designed and supervised the study. The authors express appreciation to the participants of the study for their enthusiastic support.

\section{References}

1. Hoddinott J \& Yohannes Y (2002) Dietary Diversity as a Food Security Indicator. Discussion Paper no. 136. Washington, DC: International Food Policy Research Institute, Food Consumption and Nutrition Division.

2. Hatloy A, Torheim LE \& Oshaug A (1998) Food variety: a good indicator of nutritional adequacy of the diet? A case study from an urban area in Mali, West Africa. Eur J Clin Nutr 52, 891-898.

3. Steyn N, Nel J, Nantel G, Kennedy G \& Labradarios D (2006) Food variety and dietary diversity scores: are they good indicators of dietary adequacy? Public Health Nutr $\mathbf{9}$, 644-650.

4. Mirmiran P, Azadbakht L, Esmaillzadeh A \& Azizi F (2004) Dietary diversity score in adolescents - a good indicator of the nutritional adequacy of diets: Tehran lipid and glucose study. Asia Pac J Clin Nutr 13, 56-60.

5. Mirmiran P, Azadbakht L \& Azizi F (2006) Dietary diversity within food groups: an indicator of specific nutrient adequacy in Tehranian women. $J$ Am Coll Nutr 25, 354-361.

6. Kennedy GL, Pedro MR, Seghieri C, Nantel G \& Brouwer I (2007) Dietary diversity score is a useful indicator of micronutrient intake in non-breast-feeding Filipino children. J Nutr 137, 472-477.

7. Coulston AM (2001) The search continues for a tool to evaluate dietary quality. Am J Clin Nutr 74, 417.

8. Food and Agriculture Organization of the United Nations (2001) The State of Food Security in the World 2001. Rome: FAO.

9. Azadbakht L, Mirmiran P \& Azizi F (2005) Variety scores of food groups contribute to the specific nutrient adequacy in Tehranian men. Eur J Clin Nutr 59, 1233-1240.

10. Jorde R \& Bonaa KH (2000) Calcium from dairy products, vitamin $\mathrm{D}$ intake, and blood pressure: the Tromso Study. Am J Clin Nutr 71, 1530-1535.

11. Azizi F, Mirmiran P \& Azadbakht L (2004) Predictors of cardiovascular risk factors in Tehranian adolescents: Tehran Lipid \& Glucose Study. Int J Vitam Nutr Res $\mathbf{7 4}$, 307-312.

12. Weaver CM \& Heaney RP (1998) Calcium. In Modern Nutrition in Health and Disease, 9th ed, p. 148 [ME Shils, JA Olsen, M Shike and AC Ross, editors]. Philadelphia, PA: Lippincott, Williams \& Wilkins.

13. Kant AK, Schatzkin A \& Ziegler RG (1995) Dietary diversity and subsequent cause of specific mortality in the NHANESI epidemiologic follow up study. $J$ Am Coll Nutr 14, 233-238.

14. McCollough ML, Feskanich D, Stampfer MJ, Fiovannucci EL, Rimm EB, Hu FB, Spiegelman D, Hunter DJ, Colditz GA \& Willett WC (2002) Diet quality and major chronic disease risk in men and women: moving toward improved dietary guidance. Eur J Clin Nutr 57, 930-939. 
15. Wahlqvist ML, Lo CS \& Myers KA (1989) Food variety is associated with less macrovascular disease in those with type II diabetes and their healthy controls. J Am Coll Nutr $\mathbf{8}$, 515-523.

16. Miller WL, Crabtree BF \& Evans DK (1992) Exploratory study of the relationship between hypertension and diet diversity among Saba Islanders. Public Health Rep 107, 426-432.

17. Azadbakht L, Mirmiran P, Esmaillzadeh A \& Azizi F (2006) Dietary diversity score and cardiovascular risk factors in Tehranian adults. Public Health Nutr 9, 728-736.

18. Azadbakht L, Mirmiran P \& Azizi F (2005) Dietary diversity score is favorably associated with the metabolic syndrome in Tehranian adults. Int J Obes (Lond) 29, 1361-1367.

19. Sarrafzadegan N, Baghaei AM, Sadri GH et al. (2006) Isfahan Healthy Heart Program: evaluation of comprehensive, community-based interventions for non-communicable disease prevention. Prev Control 2, 73-84.

20. Sarrafzadegan N, Sadri GH, Malekafzali H et al. (2003) Isfahan Healthy Heart Program: a comprehensive integrated community-based program for cardiovascular disease prevention and control. Design, methods and initial experience. Acta Cardiol 58, 309-320.

21. Sarraf-Zadegan N, Baghaei AM, Kelishadi R \& Boshtam M (2004) First annual evaluation of Isfahan Healthy Heart Program (IHHP): full report. http://www.ihhp.mui.ac.ir/ articles/enarchives/1stihhpreport.htm (accessed September 2007).

22. IHHP projects (2001) Healthy food for healthy community. http://ihhp.ir/ihhp/display.aspx?id=1495 (accessed September 2007).

23. Kant AK, Schatzkin A, Graubard BI \& Schairer C (2000) A prospective study of diet quality and mortality in women. JAMA 283, 2109-2115.

24. Eliakim A, Nemet D, Balakirski Y \& Epstein Y (2007) The effects of nutritional-physical activity school-based intervention on fatness and fitness in preschool children. J Pediatr Endocrinol Metab 20, 711-718.

25. Koehler S, Sichert-Hellert W \& Kersting M (2007) Measuring the effects of nutritional counseling on total infant diet in a randomized controlled intervention trial. J Pediatr Gastroenterol Nutr 45, 106-113.

26. Goulet J, Lapointe A, Lamarche B \& Lemieux S (2007) Effect of a nutritional intervention promoting the Mediterranean food pattern on anthropometric profile in healthy women from the Quebec city metropolitan area. Eur J Clin Nutr 61, 1293-1300.

27. Gadallah M, Rady M, Salem B, Aly EM \& Anwer W (2002) The effect of nutritional intervention program on the prevalence of anemia among pregnant women in rural areas of Belbis district - Sharkia Governorate - Egypt. J Egypt Public Health Assoc 77, 261-273.

\section{Appendix}

\section{Foods and food groups in the FFQ}

\begin{tabular}{|c|c|c|}
\hline Hydrogenated oil & 26. & Chocolate \\
\hline Liquid oil & 27. & Boiled potato \\
\hline Olive oil & 28. & Fried potato \\
\hline Animal oil & 29. & Fresh fruit \\
\hline Margarine & 30. & Fresh juice \\
\hline Butter & 31. & Dried fruit \\
\hline Tallow & 32. & Fresh vegetables \\
\hline Cream & 33. & Cooked vegetables \\
\hline Cheese & 34. & Dried vegetables \\
\hline High-fat milk & 35. & Pickled vegetables \\
\hline Low-fat milk & 36. & Salted vegetables \\
\hline High-fat yoghurt & 37. & Walnuts \\
\hline Low-fat yoghurt & 38. & Pistachios, almonds \\
\hline Liver, kidney, heart & 39. & Seeds \\
\hline Kalepache (a traditional Iranian food consisting of organ meats) & 40. & Commercial fruit \\
\hline Sausages & 41. & Eggs \\
\hline Red meat & 42. & Pulses \\
\hline Poultry & 43. & Soya \\
\hline Fish & 44. & Canned foods \\
\hline Bread & 45. & Fried meals \\
\hline Rice & 46. & Hamburger \\
\hline Sweet cola & 47. & Pizza \\
\hline Diet cola & 48. & Mayonnaise \\
\hline Jam & 49. & Garlic \\
\hline
\end{tabular}

